\title{
Cluster Analysis of the Yale Global Tic Severity Scale (YGTSS): Symptom Dimensions and Clinical Correlates in an Outpatient Youth Sample
}

\author{
Katharina Kircanski • Douglas W. Woods • \\ Susanna W. Chang • Emily J. Ricketts • \\ John C. Piacentini
}

Published online: 13 April 2010

(C) The Author(s) 2010. This article is published with open access at Springerlink.com

\begin{abstract}
Tic disorders are heterogeneous, with symptoms varying widely both within and across patients. Exploration of symptom clusters may aid in the identification of symptom dimensions of empirical and treatment import. This article presents the results of two studies investigating tic symptom clusters using a sample of 99 youth ( $\mathrm{M}$ age $=$ $10.7,81 \%$ male, $77 \%$ Caucasian) diagnosed with a primary tic disorder (Tourette's disorder or chronic tic disorder), across two university-based outpatient clinics specializing in tic and related disorders. In Study 1, a cluster analysis of the Yale Global Tic Severity Scale (YGTSS) identified four symptom dimensions: predominantly complex tics; simple head/face tics; simple body tics; and simple vocal/facial tics. In Study 2, these clusters were shown to be differentially associated with demographic and clinical characteristics. Findings lend support to prior research on tic phenomenology, help to organize treatment goals, and suggest symptom dimensions of tic disorders for further evaluation.
\end{abstract}

K. Kircanski ( $\square)$

Department of Psychology, University of California-Los Angeles, 1285 Franz Hall, Box 951563, Los Angeles, CA 90095-1563, USA

e-mail: kkir@ucla.edu

D. W. Woods • E. J. Ricketts

Department of Psychology, University of Wisconsin-Milwaukee, Milwaukee, WI, USA

S. W. Chang $\cdot$ J. C. Piacentini

Division of Child and Adolescent Psychiatry,

UCLA-NPI, Semel Institute for Neuroscience and Human

Behavior, University of California-Los Angeles,

Los Angeles, CA, USA
Keywords Tics · Tourette's disorder Chronic tic disorder . Symptom dimensions · Cluster analysis · Yale Global Tic Severity Scale

\section{Introduction}

Tic disorders comprise a wide range of symptoms. Individual tics, defined as "sudden, rapid, recurrent, nonrhythmic, and stereotyped" motor movements or vocalizations (American Psychiatric Association 2000, p. 108), vary both within and across patients. For example, tics may differ in type (motor or vocal), complexity (number of muscle groups involved), and degree of interference in functioning (none to severe). This diversity in tic symptomatology likely reflects a complex etiology involving genetic and environmental influences, as well as proximal neurobiological underpinnings (Conelea and Woods 2008; Himle et al. 2006; McMahon et al. 2003; Swain et al. 2007) that may differ across tic types (Aldridge et al. 2004; Wolf et al. 1996). Currently there exist several tic disorder diagnoses, although it has been argued that the two more persistent disorders, Tourette's disorder and chronic (motor or vocal) tic disorder, may share similar pathophysiology (Saccomani et al. 2005). Tics also vary in course, although they typically emerge in early childhood, peak in middle childhood, and may markedly reduce or disappear in adulthood (Leckman 2003; Leckman et al. 2006). Children and adolescents with Tourette's disorder appear at risk for learning difficulties, overall school and social impairment, and a variety of internalizing and externalizing psychiatric comorbidities (Leckman et al. 2006; Zhu et al. 2006). As youth represents the prominent life stage during which tics emerge and reach peak severity, it offers a particularly 
important period in which to better understand and treat tic disorders.

As tic disorders are no longer viewed as unitary conditions (Robertson et al. 2008) and a reliance on global symptom counts as an indicator of severity may misrepresent the diagnoses (Storch et al. 2007), numerous authors have attempted to organize the multifaceted nature of tic phenomenology along meaningful dimensions by drawing on clinical observations and growing empirical data (e.g., Freeman et al. 2000; Jagger et al. 1982; Kuperman 2002; Leckman et al. 1999; Swain and Leckman 2003). Four studies have used cluster analysis, some with subsequent factor analysis, to investigate relative associations among the symptoms of Tourette's disorder, with the notion that, across patients, symptoms classified in a particular cluster co-occur more often with one another than with variables classified in other clusters (Alsobrook and Pauls 2002; Mathews et al. 2007; Robertson and Cavanna 2007; Robertson et al. 2008). This variable-centered application of cluster analysis is distinct from cluster analytic methods used to typologize individuals, and it is often selected when factor analytic methods such as principal components analysis (PCA) are not appropriate to examine the data. Most notably, as PCA has been designed for use with continuous variables, it is typically suboptimal for use with dichotomous variables such as the presence versus absence of symptoms, and it tends to require very large sample sizes that may not be available for certain patient groups. Conversely, cluster analysis may be used with a wider range of variable types, including binary items, and sample sizes (Breakwell et al. 2006; Reise et al. 2000). Thus, cluster analysis appears to be a logical and potentially fruitful method of classifying tic disorder symptoms; if symptom clusters were to indicate distinct diagnostic or functional dimensions of tics, these may allow for more meaningful analyses of symptom-specific clinical correlates and help organize assessment and treatment procedures more effectively around groups of tics (Swain and Leckman 2003). For example, cluster analysis findings may suggest ways in which behavioral treatments could be better structured for particular sensory experiences, domains of impairment, or comorbid problems associated with particular groups of tics, potentially enhancing treatment effectiveness and efficiency.

In the first cluster analysis of tic symptomatology, Alsobrook and Pauls (2002) studied 85 child and adult probands diagnosed with Tourette's disorder using items from the Schedule for Tourette and Other Behavioral Syndromes (Pauls and Hurst 1981). Twelve symptom clusters, ranging in size from one to four items, were then subjected to principal components analysis (PCA). The PCA results indicated four symptom factors, including aggressive behaviors, pure motor and vocal tics, compulsive behaviors, and tapping/absence of grunting. The aggressive factor was positively associated with comorbid attention-deficit/hyperactivity disorder (ADHD) in probands and first-degree relatives, and the compulsive factor was positively associated with earlier age of tic disorder onset and comorbid ADHD in probands. However, the results should be interpreted with caution, as binary oneitem clusters are not typically suitable for PCA.

Mathews et al. (2007) gathered lifetime data for children and adults diagnosed with Tourette's disorder from two genetically isolated samples using the Yale Self Report Form (YSRF; Tourette Syndrome Association International Consortium for Genetics 1999). In each sample, they found evidence for two symptom clusters, a simple tic cluster and complex tic cluster, and that the complex tic cluster was positively associated with tic severity, impairment, and comorbid obsessive compulsive disorder (OCD) symptoms. As these results were based on lifetime rather than current tic symptomatology, participant data may have been biased by limits in retrospective self-report, and tics forming the clusters may not have been concurrently present.

Robertson and Cavanna (2007) also studied child and adult participants, 69 of whom exhibited tic and related symptoms using the National Hospital Interview Schedule for Gilles de la Tourette Syndrome (NHIS-GTS; Robertson and Eapen 1996), although they did not necessarily meet criteria for a tic disorder. Additionally, several of the items included non-tic-specific phenomena (e.g., "anxiety"). The obtained cluster sizes ranged from one to six items, and when subjected to PCA, only one factor comprised pure tics. These factors were not studied in their associations with other clinical criteria. Subsequently, in a larger sample of 410 children and adults diagnosed with Tourette's disorder using the NHIS-GTS, Robertson et al. (2008) identified seven tic-specific symptom clusters that were subjected to PCA. Five factors were obtained and defined as socially inappropriate behaviors/complex vocal tics, complex motor tics, simple tics, compulsive behaviors, and touching self. Positive associations were shown between the first and third factors and comorbid ADHD, and the first four factors and comorbid OCD. Again these results should be interpreted with caution, as several one-item clusters were used in the PCA, and the compulsive factor included broader items (e.g., "leg and foot movement").

In sum, the results of prior cluster analytic studies examining the symptom structure of Tourette's disorder have been mixed. Across two studies, symptoms separated along dimensions of simple versus complex tics (Mathews et al. 2007; Robertson et al. 2008), and a compulsive behaviors classification was obtained (Alsobrook and Pauls 2002; Robertson et al. 2008). Symptom groupings differed across the studies, and each had aforementioned methodological limitations. Additionally, no prior studies have been restricted to samples of youth, when tics are typically most 
prominent and multifaceted, or have included diagnoses of chronic tic disorder, which may share common pathophysiology with Tourette's disorder (Saccomani et al. 2005).

The overall goals of the two current studies were to investigate symptom dimensions of chronic tic disorders and their clinical correlates in an outpatient sample of youth with either Tourette's disorder or chronic motor tic disorder, utilizing symptom-level data from the Yale Global Tic Severity Scale (YGTSS; Leckman et al. 1989). The YGTSS is a clinician-administered measure of tic symptomatology with the ability to gather comprehensive, concurrent data across multiple tic disorder diagnoses. As such, it may serve as a particularly useful measure of individual tic symptoms to subject to cluster analysis in an investigation of possible symptom dimensions. Such an analysis of the YGTSS symptom-level data has not been conducted to date.

\section{Study 1: Cluster Analysis}

The aim of Study 1 was to utilize cluster analysis as the primary method of examining tic disorder symptom dimensions, based on its use in prior studies and utility for dichotomous symptom data. With regard to study hypotheses, we expected that tic disorder symptom clusters would separate along dimensions of simple versus complex tics, based on two prior findings (Mathews et al. 2007; Robertson et al. 2008). Similar to the previous cluster analytic studies (Alsobrook and Pauls 2002; Mathews et al. 2007; Robertson and Cavanna 2007; Robertson et al. 2008), we made no other a priori assumptions regarding symptom clusters.

\section{Method}

\section{Participants}

The sample consisted of a series of youth (total $N=99$ ) ages 7 to $17(M=10.74, S D=2.41)$, who were referred to one of two outpatient clinics specializing in the assessment and/or treatment of childhood and adolescent tic and related disorders. The two sites were the UCLA Child OCD, Anxiety, and Tic Disorders Program $(n=76)$ and the UWM Tic Disorder Specialty Clinic $(n=23)$. All youth met Diagnostic and Statistical Manual of Mental Disorders (4th edition, text revision; DSM-IV-TR; APA 2000) diagnostic criteria for a primary tic disorder, including Tourette's disorder $(n=88)$ and chronic motor tic disorder $(n=$ 11 ), as determined by clinical interview with doctoral-level psychologists or doctoral students in clinical psychology supervised by licensed psychologists. Interviewers received a training period involving careful read-through of the study measures, observation and corating of videotaped administrations of each measure, corating of at least one live interview, and matching on diagnoses with an experienced senior clinician. Interviewers received weekly supervision on each case from an experienced senior clinician at their site (D.W., UWM; S.C., UCLA). No youth met criteria for chronic vocal tic disorder, consistent with previous findings of the relatively low prevalence of this diagnosis (Shapiro et al. 1988). Most participants were involved in ongoing research projects with the remainder seeking clinical services. At intake, all youth completed an informed assent following an informed consent with their parent/guardian to use the data from the YGTSS and additional assessment measures for research purposes. Sample demographics were $80.8 \%$ male, and $77.1 \%$ Caucasian, $5.7 \%$ Latino/a, $4.3 \%$ Asian/Pacific Islander, 2.9\% African-American, and 10.0\% mixed. Parent/guardian mean household income was in the $\$ 60,000$ to $\$ 80,000$ range. The two sites did not differ on any demographic variables.

\section{Measures}

The Yale Global Tic Severity Scale (YGTSS; Leckman et al. 1989) was jointly administered to each youth and parent/ guardian by direct inquiry, including the symptom checklist, Total Tic Score items, and Impairment Score item, as part of a larger assessment battery completed by all youth and families evaluated in the programs and approved by the universities' Institutional Review Boards. The YGTSS symptom checklist lists 46 tic disorder symptoms, including 12 simple motor tics (e.g., eye blinking), 19 complex motor tics (e.g., facial expressions), seven simple vocal tics (e.g., coughing), and eight complex vocal tics (e.g., words), with four of these items designated on the instrument as "other" symptoms. For the purposes of the current analyses, where possible, the symptom checklist items endorsed as "other" were given new designations based on symptom commonalities across participants, including "torso movements" in the simple motor category and "breath-related sounds" in the simple vocal category. The remaining two "other" items, one in the complex motor category and one in the simple vocal category, were subsequently defined as "miscellaneous".

A 50-point Total Tic Score is comprised of 10 items that are anchored by the symptoms identified on the checklist and scored separately for motor tics and vocal tics on 5point Likert scales corresponding to tic number, frequency, intensity, complexity, and interference. Factor analyses of the Total Tic Score items have demonstrated good convergent and discriminant validity and inter-rater reliability in child and adolescent samples (Leckman et al. 1989; Storch et al. 2005, 2007; Walkup et al. 1992). Several studies have also demonstrated associations between Total 
Tic Score items and other clinical characteristics, including positive correlations between YGTSS tic severity and school impairment, thought problems, aggressive behavior, delinquent behavior, and lower social competence (Zhu et al. 2006), YGTSS tic complexity and lower functional competence even when controlling for psychiatric comorbidity (Himle et al. 2007), and in children ages 10 and older, but not younger, between YGTSS tic severity, complexity, number, and interference and experience of premonitory urges, or unpleasant sensory phenomenon preceding tics (Woods et al. 2005).

\section{Statistical Analyses}

All analyses were conducted using SPSS Version 16.0. Data for the two sites were analyzed simultaneously. The sites did not differ with regard to any YGTSS summary scores. As in the previous cluster analytic studies (Alsobrook and Pauls 2002; Mathews et al. 2007; Robertson and Cavanna 2007; Robertson et al. 2008), we used agglomerative hierarchical cluster analysis to form symptom clusters. In performing the analysis, individual tic symptoms from the YGTSS checklist were grouped into clusters using Ward's linkage method for binary data (Mathews et al. 2007). The agglomeration stages were displayed visually as a dendrogram, with the cluster formations plotted along a scaled between-stage distance axis. Using the same procedures as the prior studies, visual inspection of the dendrogram according to a series of decision rules was used to determine the appropriate formations of clusters. Tic symptoms were classified into a cluster when: (a) their dendrogram lines converged within a 10 -unit window on the dendrogram cluster distance axis; and (b) the convergence occurred before $50(0=$ individual symptoms, $100=$ unitary cluster of all symptoms). For each of the resulting clusters, participants received a score corresponding to the number of symptoms they endorsed on that particular cluster, and these cluster scores were then used in analyses of cluster membership. Cluster scores were transformed into z-scores by subtracting the mean and dividing by the standard deviation for each variable, and these z-scores were then used in analyses of cluster correlations.

\section{Results}

The rescaled dendrogram displaying the results of the agglomerative hierarchical cluster analysis is displayed in Fig. 1, indicating the relative distances or relatedness among tic disorder symptoms. As a cluster analysis of symptoms, the clusters denote symptoms that tended to cooccur with one another more strongly than with other symptoms across patients. Four tic disorder symptom clusters were identified, which ranged from relatively heterogeneous to relatively homogeneous in symptom makeup. Cluster 1, defined as "predominantly complex tics", was composed of 18 complex motor tics (disinhibited behavior; copropraxia; self-abusive behavior; writing tics; bending or gyrating; blocking; mouth movements; rotating; eye movements; hand movements; shoulder movements; arm movements; facial movements; head gestures or movements; dystonic postures; leg, foot, or toe movements; paroxysms of tics; miscellaneous), eight complex vocal tics (disinhibited speech; coprolalia; syllables; words; speech atypicalities; palalalia; blocking; echolalia), two simple motor tics (abdominal tensing; torso movements), and three simple vocal tics (animal or bird noises; coughing; miscellaneous). Cluster 2, defined as "simple head/face tics", was composed of four simple motor tics (eye blinking; eye movements; head jerks or movements; mouth movements). Cluster 3, defined as predominantly "simple body tics", was composed of four simple motor tics (arm movements; hand movements; leg, foot, or toe movements; shoulder movements) and one complex motor tic (tic-related compulsive behaviors). Last, Cluster 4, defined as "simple vocal/facial tics", was composed of four simple vocal tics (breath-related sounds; sniffing; grunting; throat clearing) and two simple motor tics (facial grimace; nose movements).

Descriptive statistics for the obtained symptom clusters are presented in Table 1. A chi-square analysis was conducted for participant membership across the four clusters, operationalized as endorsing at least one tic on that cluster and compared to the average of membership across the clusters $(81.5 \%)$. Membership on Cluster 2 was significantly higher than average, $\chi^{2}(1)=13.728, p<0.001$, and membership on Cluster 3 was significantly lower than average, $\chi^{2}(1)=25.960, p<0.001$. Neither membership on Cluster $1, \chi^{2}(1)=0.736$, ns, nor Cluster $4, \chi^{2}(1)=1.893$, ns, was significantly different from average.

Also as shown in Table 1, cluster scores were significantly intercorrelated, with the exception of Clusters 2 and 4. Descriptive statistics for overall cluster membership are presented in Table 2. The majority of participants $(52.5 \%)$ endorsed at least one tic on all four clusters.

Pearson bivariate correlations between cluster scores and YGTSS Total Tic Score items are presented in Table 3. Cluster scores were generally highly correlated with Total Tic Score items, with the exception of Cluster 2 and YGTSS vocal tic number, frequency, and intensity, and Cluster 4 and YGTSS motor tic intensity and interference.

\section{Study 2: Clinical Correlates}

The aim of Study 2 was to evaluate functional differences among the clusters obtained in Study 1, utilizing data 


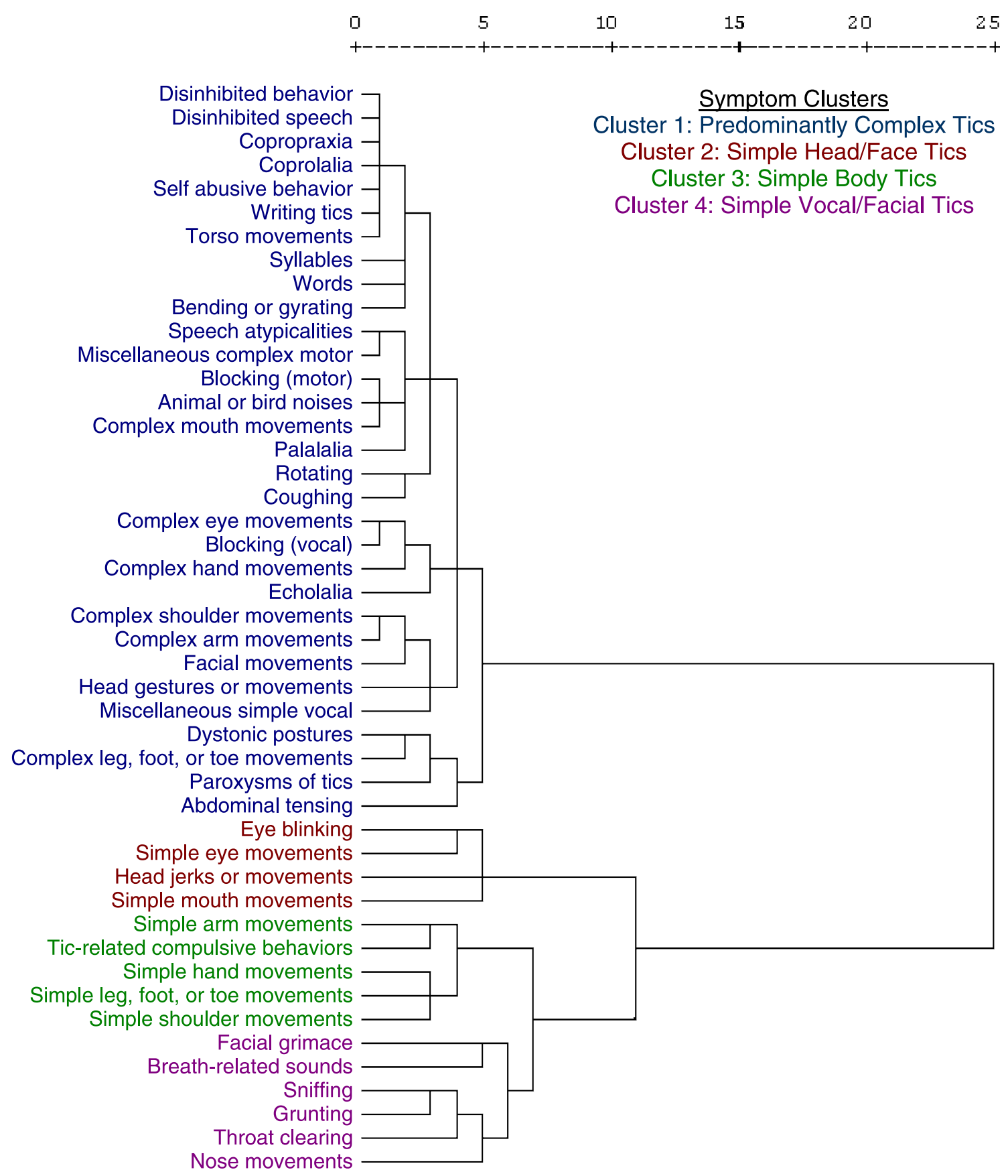

Fig. 1 Rescaled dendrogram of agglomerative hierarchical cluster analysis using Ward's method of 46 YGTSS symptom checklist items from 99 primary tic disorder participants from two sites (UCLA and UWM). Text colors indicate clustered symptoms. Symptoms were

related to various external validators including demographic variables, clinical measures, duration of illness, and psychiatric comorbidity. We had several broad hypotheses, extrapolating from prior cluster analyses and the extant literature on tic disorder phenomenology. We expected that the simple head/face and simple vocal/facial tic clusters would show a curvilinear relationship with age, peaking in middle childhood (Leckman 2003; Leckman et al. 2006), classified into a cluster when: (a) their dendrogram lines converged within a 10-unit window on the dendrogram cluster distance axis; and (b) the convergence occurred before 50 ( $0=$ individual symptoms, $100=$ unitary cluster of all symptoms)

although scores on the simple body tic cluster and complex tic cluster would continue to increase in adolescence (Jagger et al. 1982; Leckman et al. 2006). We expected that scores on the complex tics cluster would be uniquely associated with the experience of premonitory urges (Woods et al. 2005), greater concurrent obsessive-compulsive symptoms and overall clinical impairment, and poorer school performance (Himle et al. 2007; Mathews et al. 2007). We 
Table 1 Descriptive Statistics for Symptom Clusters

\begin{tabular}{llll}
\hline Variable & $\begin{array}{l}\text { Cluster 1: Predominantly } \\
\text { complex tics }\end{array}$ & $\begin{array}{l}\text { Cluster 2: Simple } \\
\text { head/face tics }\end{array}$ & $\begin{array}{l}\text { Cluster 3: Simple } \\
\text { body tics }\end{array}$ \\
\hline $\begin{array}{l}\text { Cluster membership } \\
\text { Participants with at least one tic_no. (\%) }\end{array}$ & $84(84.8)^{\mathrm{b}}$ & $95(96.0)^{\mathrm{a}}$ & $61(61.6)^{\mathrm{c}}$ \\
$\begin{array}{l}\text { Cluster score intercorrelations } \\
\text { Cluster 1 }\end{array}$ & 1.00 & & $86(86.9)^{\mathrm{b}}$ \\
Cluster 2 & $0.280^{* *}$ & 1.00 & \\
Cluster 3 & $0.407^{* * *}$ & $0.372^{* * *}$ & 1.00 \\
Cluster 4 & $0.307^{* * *}$ & 0.161 & $0.216^{*}$ \\
\hline
\end{tabular}

${ }^{*} p<0.05,{ }^{* *} p<0.01,{ }^{* * *} p<0.005$. Values in a given row with different superscripts differ significantly at $p<0.05$

also expected that scores on the complex tics cluster would be associated with longer duration of illness and greater comorbidity in youth (Alsobrook and Pauls 2002; Mathews et al. 2007; Robertson et al. 2008). As the first study to examine these specific symptom clusters, we made no other a priori assumptions regarding their clinical correlates.

\section{Method}

\section{Measures}

Each youth and parent/guardian jointly completed a series of additional assessment measures as part of the larger battery, with sample sizes indicated below. Missing forms resulted from incomplete administration of the assessment battery due to time or other logistic constraints. A series of independent samples $t$-tests indicated no significant differences between youth who completed and youth who did not complete each measure in age, cluster scores, or YGTSS summary scores.

Table 2 Overall Cluster Membership

\begin{tabular}{lc}
\hline Cluster(s) & Participants-no. (\%) \\
\hline Clusters $1,2,3$, and 4 & $52(52.5)$ \\
Clusters 1,2 , and 4 & $23(23.2)$ \\
Cluster 2 only & $6(6.1)$ \\
Clusters 2 and 4 & $5(5.1)$ \\
Clusters 1,2 , and 3 & $4(4.0)$ \\
Clusters 1,3 , and 4 & $2(2.0)$ \\
Clusters 2,3 , and 4 & $2(2.0)$ \\
Clusters 1 and 2 & $2(2.0)$ \\
Clusters 1 and 4 & $1(1.0)$ \\
Clusters 2 and 3 & $1(1.0)$ \\
Cluster 4 only & $1(1.0)$ \\
\hline
\end{tabular}

Cluster 1 Predominantly Complex Tics; Cluster 2 Simple Head/Face Tics; Cluster 3 Simple Body Tics; Cluster 4 Simple Vocal/Facial Tics

\section{Clinical Measures}

YGTSS Impairment Score (Leckman et al. $1989 ; n=99$ ) is a 50-point Likert scale representing impairment in functioning specific to tic disorder symptoms.

Child Yale-Brown Obsessive-Compulsive Scale (CYBOCS; Scahill et al. $1997 ; n=71$ ) is a clinicianadministered semi-structured interview that provides a measure of obsessive-compulsive symptomatology, which is commonly found to be associated with tic disorders in youth (Grados et al. 2001; Pauls et al. 1986). The CYBOCS has good reliability and validity (Scahill et al. 1997; Storch et al. 2004).

Child Behavior Checklist (CBCL; Achenbach, 1991; $n=$ 67) assesses behavioral and clinical domains of youth internalizing and externalizing problems, academic achievement, and social competence, and has demonstrated good validity, internal consistency, and test-retest reliability (Achenbach and Rescorla 2001). Parent t-scores were utilized.

School performance $(n=65)$ was measured using a fourpoint Likert scale representing overall academic performance (failing, below average, average, above average).

Premonitory Urge for Tics Scale (PUTS; Woods et al., $2005 ; n=42$ ) measures the experience of premonitory urges preceding tics in child and adolescent samples, and possesses sound psychometrics as reported by the authors.

Duration of Illness

Number of years of illness duration $(n=76)$ was assessed as part of the parent/guardian interview administered at both sites.

Psychiatric Comorbidity

Anxiety Disorders Interview Schedule for DSM-IV: Child and Parent Versions (ADIS-IV; Silverman and Albano 1996; $n=48$ ) or Kiddie-Schedule for Affective Disorders 
Table 3 Correlations Between Cluster Scores and YGTSS Summary Scores

\begin{tabular}{|c|c|c|c|c|}
\hline Summary Score & $\begin{array}{l}\text { Cluster 1: Predominantly } \\
\text { complex tics }\end{array}$ & $\begin{array}{l}\text { Cluster 2: Simple } \\
\text { head/face tics }\end{array}$ & $\begin{array}{l}\text { Cluster 3: Simple body } \\
\text { tics }\end{array}$ & $\begin{array}{l}\text { Cluster 4: Simple vocal/facial } \\
\text { tics }\end{array}$ \\
\hline \multicolumn{5}{|l|}{ Motor } \\
\hline Number & $0.634 * * *$ & $0.395 * * *$ & $0.462 * * *$ & $0.271 * *$ \\
\hline Frequency & $0.345^{* * *}$ & $0.393 * * *$ & $0.360 * * *$ & $0.348 * * *$ \\
\hline Intensity & $0.511 * * *$ & $0.428 * * *$ & $0.258^{*}$ & 0.126 \\
\hline Complexity & $0.638 * * *$ & $0.282 * *$ & $0.391 * * *$ & $0.271 * *$ \\
\hline Interference & $0.540 * * *$ & $0.314 * * *$ & $0.400 * * *$ & 0.117 \\
\hline \multicolumn{5}{|l|}{ Vocal } \\
\hline Number & $0.716^{* * *}$ & 0.204 & $0.361 * * *$ & $0.577 * * *$ \\
\hline Frequency & $0.411 * * *$ & 0.136 & $0.311 * * *$ & $0.574 * * *$ \\
\hline Intensity & $0.572 * * *$ & 0.196 & $0.374 * * *$ & $0.562 * * *$ \\
\hline Complexity & $0.681 * * *$ & $0.251^{*}$ & $0.291 * *$ & $0.267^{*}$ \\
\hline Interference & $0.554 * * *$ & $0.232 *$ & $0.342 * * *$ & $0.458 * * *$ \\
\hline
\end{tabular}

${ }^{*} p<0.05,{ }^{*} p<0.01,{ }^{* * *} p<0.005$. No correction was applied for multiple statistical testing. YGTSS Yale Global Tic Severity Scale

and Schizophrenia (K-SADS-PL; Kaufman et al. 1997; $n=$ 35) was used to gather data on diagnosis of coexisting disorders. ADIS-IV is a semistructured diagnostic interview that assesses mood, anxiety, and externalizing disorders experienced by children and adolescents, and demonstrates good test-retest reliability (Silverman et al. 2001) and concurrent validity (Wood et al. 2002). K-SADS-PL is a semistructured diagnostic interview that assesses major Axis I disorders in childhood and adolescence and possesses sound psychometrics (Kaufman et al. 1997).

\section{Statistical Analyses}

All analyses were conducted using SPSS Version 16.0. Data for the two sites were analyzed simultaneously. A series of pearson bivariate correlational analyses were conducted between youth cluster z-scores and various external validity criteria. Cases were excluded for a particular analysis when they contained missing data. Correlational analyses of clinical characteristics partialed age in the event of differential cluster associations with age (Leckman 2003; Leckman et al. 2006), with the exception of CBCL t-scores designed to control for age. In order to examine the clusters' associations with specific comorbid diagnoses, a series of logistic regressions were performed in which each cluster z-score served as a predictor of the presence versus absence of each comorbid diagnosis.

\section{Results}

Figure 2 displays associations between the four cluster zscores and age, with lines representing quadratic trends. A series of regression analyses did not yield a significant relationship between scores on Cluster 1 and age in either linear $\left(R^{2}=0.015, b_{1}=0.050, \mathrm{~ns}\right)$ or quadratic $\left(R^{2}=\right.$ $\left.0.015, b_{1}=0.032, b_{2}=0.001, \mathrm{~ns}\right)$ models, scores on Cluster 2 in either linear $\left(R^{2}=0.000, b_{1}=-0.006, \mathrm{~ns}\right)$ or quadratic $\left(R^{2}=0.010, b_{1}=0.307, b_{2}=-0.014, \mathrm{~ns}\right)$ models, and scores on Cluster 3 in either linear $\left(R^{2}=0.020, b_{1}=0.058, \mathrm{~ns}\right)$ and quadratic $\left(R^{2}=0.020, b_{1}=0.024, b_{2}=0.002, \mathrm{~ns}\right)$ models. Scores on Cluster 4 were not predicted by age in the linear model $\left(R^{2}=0.007, b_{1}=-0.034, \mathrm{~ns}\right)$, but were predicted by age in the quadratic model $\left(R^{2}=0.058, b_{1}=0.675, b_{2}=-0.031\right.$, $p<0.05$ one-tailed). A series of point biserial correlations conducted between cluster scores and gender were nonsignificant.

Table 4 presents associations between cluster scores and clinical characteristics. Cluster 1 scores were positively associated with premonitory urge measured by the PUTS total score, while Cluster 1 and Cluster 3 scores were positively associated with CYBOCS Total Score. Clusters 1, 2 , and 3 scores were positively associated with YGTSS Impairment. Each cluster evidenced a unique pattern of association with the CBCL broad-band and specific subscale scores. Only Cluster 2 scores were negatively associated with school performance as measured by both the CBCL school subscale and school performance measure. Cluster 1 scores were also positively associated with number of concurrent diagnoses and demonstrated a trend toward a positive association with duration of illness $(p=0.06)$.

Table 5 presents the results of a series of logistic regressions predicting diagnoses of specific coexisting disorders from cluster scores, partialing patient age. Cluster 1 scores were uniquely associated with concurrent diagnosis of OCD, $\beta=0.714, p<0.05$, but none of the other diagnoses examined. There were no significant relationships between any of the remaining clusters and concurrent diagnoses. 


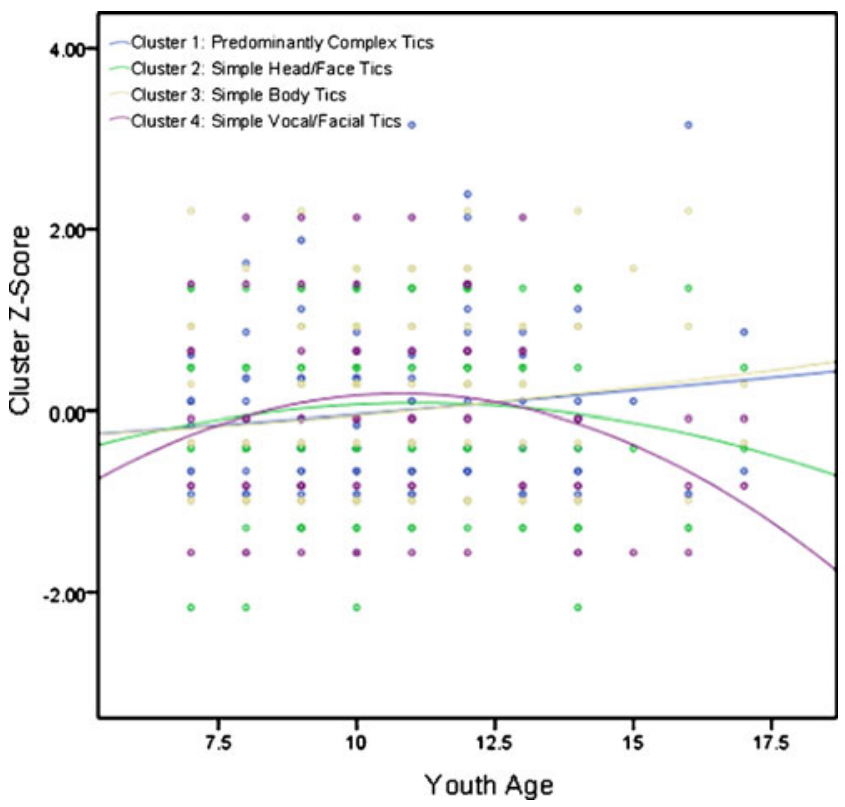

Fig. 2 Lines represent quadratic trends

\section{Discussion}

The present research represents the first investigation of tic disorder symptom clusters and their clinical correlates in a clinical sample of youth with primary Tourette's disorder or chronic tic disorder. Overall, findings suggest that the tic symptoms experienced by youngsters may be differentially associated with one another and with external validity criteria of empirical and treatment import.

Associations among Tic Disorder Symptoms

Study 1 results indicated differential associations among tic disorder symptoms. First, as predicted, the obtained symptom clusters separated along dimensions of simple versus complex tics, as found in two prior cluster analyses (Mathews et al. 2007; Robertson et al. 2008). Cluster 1 comprising predominantly complex tics was rather large and diverse, as it included motor and vocal tics that were generally characterized by movements or vocalizations of relative intricacy and/or duration. Although Cluster 1 was

Table 4 Correlations Between Cluster Scores and Clinical Characteristics

\begin{tabular}{|c|c|c|c|c|}
\hline Measure & $\begin{array}{l}\text { Cluster 1: Predominantly } \\
\text { complex tics }\end{array}$ & $\begin{array}{l}\text { Cluster 2: Simple } \\
\text { head/face tics }\end{array}$ & $\begin{array}{l}\text { Cluster 3: Simple } \\
\text { body tics }\end{array}$ & $\begin{array}{l}\text { Cluster 4: Simple } \\
\text { vocal/facial tics }\end{array}$ \\
\hline PUTS total score ${ }^{\mathrm{a}}$ & $0.401 *$ & 0.162 & 0.130 & -0.091 \\
\hline CYBOCS total score ${ }^{a}$ & $0.313^{* *}$ & 0.021 & $0.292 *$ & 0.033 \\
\hline YGTSS impairment $^{\mathrm{a}}$ & $0.575 * * *$ & $0.381 * * *$ & $0.281 * *$ & 0.193 \\
\hline \multicolumn{5}{|l|}{ CBCL parent T-score } \\
\hline Internalizing & $0.381 * * *$ & 0.141 & 0.205 & 0.198 \\
\hline Externalizing & $0.358 * * *$ & 0.135 & 0.147 & 0.208 \\
\hline Activities & -0.231 & $-0.326^{* *}$ & $-0.376 * * *$ & 0.018 \\
\hline Social & $-0.298^{*}$ & -0.061 & -0.129 & -0.205 \\
\hline School & -0.115 & $-0.285^{*}$ & -0.161 & -0.006 \\
\hline Total competence & $-0.275^{*}$ & -0.201 & $-0.301 *$ & -0.102 \\
\hline Withdrawal & $0.259 *$ & 0.164 & 0.108 & -0.012 \\
\hline Somatic complaints & $0.436 * * *$ & 0.183 & $0.308^{*}$ & $0.326^{* *}$ \\
\hline Anxiety/Depression & $0.426 * * *$ & 0.026 & 0.159 & 0.154 \\
\hline Social problems & $0.336^{* *}$ & $0.259 *$ & 0.127 & 0.065 \\
\hline Thought problems & $0.247 *$ & -0.018 & 0.018 & 0.120 \\
\hline Attention problems & $0.354 * * *$ & $0.249^{*}$ & 0.171 & 0.213 \\
\hline Delinquent behavior & 0.189 & 0.127 & 0.216 & 0.128 \\
\hline Aggressive behavior & $0.327 * *$ & 0.060 & 0.112 & 0.144 \\
\hline School performance ${ }^{\mathrm{a}}$ & -0.192 & $-0.343 * *$ & -0.085 & 0.004 \\
\hline Duration of illness in years ${ }^{\mathrm{a}}$ & 0.217 & 0.107 & -0.046 & 0.188 \\
\hline Number of concurrent diagnoses ${ }^{\mathrm{a}}$ & $0.333 * * *$ & 0.085 & 0.075 & 0.163 \\
\hline
\end{tabular}

CBCL Child Behavior Checklist; CYBOCS Child Yale-Brown OC Scale; PUTS Premonitory Urge for Tics Scale; YGTSS Yale Global Tic Severity Scale

${ }^{\text {a }}$ Correlations partial participant age. No correction was applied for multiple statistical testing

${ }^{*} p<0.05, * * p<0.01, * * * p<0.005$ 
Table 5 Cluster Scores Predicting Diagnoses of Coexisting Disorders, Partialing Patient Age

\begin{tabular}{lcccc}
\hline Variable & $\begin{array}{l}\text { Cluster 1: Predominantly } \\
\text { complex tics }\end{array}$ & $\begin{array}{l}\text { Cluster 2: Simple } \\
\text { head/face tics }\end{array}$ & $\begin{array}{l}\text { Cluster 3: Simple } \\
\text { body tics }\end{array}$ & $\begin{array}{l}\text { Cluster 4: Simple } \\
\text { vocal/facial tics }\end{array}$ \\
\hline $\begin{array}{l}\text { Diagnosis of Coexisting Disorder } \\
\text { a }\end{array}$ & & & & -0.180 \\
OCD & $0.741^{*}$ & -0.291 & -0.361 & -0.044 \\
ADHD & 0.452 & 0.192 & -0.108 & 0.152 \\
Anxiety disorder & 0.221 & 0.475 & 1.407 & 0.252 \\
Major depression & 1.273 & -1.285 & 0.174 \\
\hline
\end{tabular}

$A D H D$ Attention-Deficit/Hyperactivity Disorder; $O C D$ Obsessive-Compulsive Disorder

${ }^{a}$ Values represent $\beta$ coefficients in logistic regression. No correction was applied for multiple statistical testing $* p<0.05$

relatively heterogeneous, the results suggest that the multiple complex tics and several simple tics within this cluster tend to co-occur or may be related. For example, the two simple tics falling on this cluster, abdominal tensing and torso movements, involve relatively large muscle groups, and may be associated with complex motor tics such as bending or gyrating that involve similar muscle groups. Interestingly, Clusters 2, 3, and 4 comprised different types of simple tics, a finding not evidenced in prior cluster analytic studies of mixed youth and adult samples (Alsobrook and Pauls 2002; Mathews et al. 2007; Robertson and Cavanna 2007; Robertson et al. 2008). The simple tic clusters in the current study also differentiated between motor and vocal tics. Further, the two simple motor tic clusters differentiated between location, with one cluster involving movements of the head and face (eyes and mouth) and the other involving movements of the body (shoulder, arm, hand, leg/foot/toe, and compulsive behaviors). These findings suggest that simple tics in youth may sort more specifically by type and location, whereas complex tics may be more heterogeneous in nature.

Additionally, Study 1 results for participant membership across the clusters indicated that simple head/face tics (Cluster 2) were highly common. This result aligns with previous proposals that the onset of tic symptoms often begins with simple movements of the head and face (Leckman et al. 2006), and thus may be more prevalent in youth samples. The relative frequency of predominantly complex tics (Cluster 1) also highlights the regularity of more elaborate and protracted tics in youngsters, which may provide useful phenomenological information when planning tic assessment and treatment with youth. Simple body tics (Cluster 3) appeared slightly less common than the other tic clusters with regard to participant membership. However, on average, participants endorsed at least one tic from this cluster.

Likewise, the data regarding cluster intercorrelations indicated a generally high degree of association among the clusters, similar to one prior study (Robertson and Cavanna
2007) and of greater strength than in other prior studies (Alsobrook and Pauls 2002; Mathews et al. 2007; Robertson et al. 2008). Additionally, it should be noted that the majority of youth in the current study endorsed at least one tic on all four symptom clusters, exhibiting substantial overlap in overall cluster membership. As tics are typically most prominent in youth and tend to decrease in adulthood (Leckman 2003; Leckman et al. 2006), it is possible that the stronger associations among symptoms observed in the current study may be related to greater power to detect symptom associations in youth. Additionally, such strong associations may have been influenced by tic severity reaching its peak at the time of seeking treatment. Correlations between cluster scores and YGTSS summary scores also indicated shared symptomatic variance across the measures. However, these data should be interpreted within the context of Study 2 findings for the clusters' differential associations with external validity criteria.

\section{External Validity of Symptom Clusters}

Study 2 results revealed discriminative validity of the obtained symptom clusters with regard to external validators. First, with regard to sample demographics, simple facial/vocal tics (Cluster 4) exhibited a significant curvilinear relationship with age, peaking in middle childhood and decreasing in adolescence as predicted, and simple head/ face tics (Cluster 2) exhibited a trend toward this effect. This relationship was not shown for simple body tics (Cluster 3) and predominantly complex tics (Cluster 1), which appeared to increase in adolescence as predicted, albeit non-significantly. These findings lend a degree of empirical support to prior suggestions that tic symptoms begin with simple movements of the head and face (Leckman 2003; Leckman et al. 2006), decreasing over time as tics progress in a cephalo-caudal fashion down the body (Jagger et al. 1982) and toward more complex tics (Leckman et al. 2006). Findings for age may also help to educate families about expectations for tic course and 
development. In future research, findings would need to be substantiated through within-person longitudinal analyses.

Findings for clinical variables also generally aligned with Study 2 predictions and highlighted the significance of complex tics. Predominantly complex tics (Cluster 1) exhibited a unique association with premonitory urges while controlling for age. Although it has been proposed that age is a critical factor in the development of premonitory urges (Banaschewski et al. 2003), the present results are consistent with the notion that tic complexity may also play an important role (Woods et al. 2005). As a consequence, treatment of complex tics may particularly benefit from an emphasis on reducing experience of or habituating to unpleasant premonitory sensations (Himle et al. 2006). Predominantly complex tics (Cluster 1) were further associated with poorer functioning across CBCL domains including externalizing, internalizing, somatic, cognitive, and social problems, obsessive-compulsive symptoms, and multimorbidity. These data highlight potential complicating and risk factors associated with more complex tics (Himle et al. 2007; Mathews et al. 2007), which may have implications for tic assessment and/or treatment. For instance, assessment of complex tics may involve more frequent differential diagnosis between tics and externalizing or aggressive behaviors, and treatment for the majority of youngsters who exhibit at least one complex tic may need to incorporate a wide range of tic-related problem areas. Planning and expectations for treatment may also benefit from a comprehensive and multi-strategy approach.

Less anticipated were findings that simple head/face tics (Cluster 2) seemed uniquely predictive of school functioning as measured by the CBCL and school performance scale. It is plausible that these types of tics (e.g., eye movements, head jerks) may be particularly disruptive in classroom settings where visual concentration on material is critical (Leckman et al. 2006; Walkup et al. 1992), and treatment goals for this group of tics may focus on reducing school interference.

Simple body tics (Cluster 3) were associated with fewer, albeit significant, external validators, including obsessivecompulsive symptoms as measured by the CYBOCS but not as indicated by concurrent diagnosis of OCD. These findings are somewhat consistent with the inclusion of compulsive behaviors on this cluster, and again suggestive of the need for careful differential diagnosis between tics and related conditions. Treatment goals may also incorporate helping patients to distinguish tics from compulsive behaviors or to extend behavioral strategies across both types of symptoms. Simple vocal/tics (Cluster 4) were associated with the fewest external validators, suggesting that they pose less clinical risk, although these tics often progress to more complex vocal tics with age (Leckman et al. 2006). Further studies of tic symptom clusters may benefit from a longitudinal series of assessment points from which to examine these questions.

\section{Limitations}

It should be noted that agglomerative hierarchical cluster analysis does not utilize a model fit statistic, which limited the comparison of other possible cluster solutions, although it allowed the current findings to be compared with the previous cluster analyses of tic disorder symptoms that used the same procedures. Also similar to previous research and given our relatively focused hypotheses, we did not adjust for multiple testing. It should be noted that certain external validity criteria, including gender, were not associated with any of the symptom clusters. The sample for the current study was predominantly male, similar to the approximately 4:1 male:female ratio observed in larger studies (Freeman et al. 2000), which may have made it difficult to capture differential relations with gender. Additionally, the somewhat restricted range of the simple tic clusters (Clusters 2, 3 , and 4) and missing data for clinical characteristics may have limited the power to detect associations, although the simple tic clusters were nevertheless associated with a unique pattern of clinical variables. A more general limitation was the moderate size of the current sample. Although larger than two previous studies (Alsobrook and Pauls 2002; Robertson and Cavanna 2007) despite being restricted to youth, this sample size may have limited statistical power to detect differential cluster associations with variables such as specific psychiatric comorbidities. Future studies may utilize internet-based (e.g., Freeman et al. 2000) or internet-assisted measures in order to better evaluate such associations.

\section{Implications and Future Directions}

The current research is the first to utilize YGTSS symptomlevel data to investigate symptom dimensions of tic disorders. The results presented herein reinforce its utility for this purpose, proposing that in children and adolescents tic disorder symptoms cluster differently with one another and with criteria of prognostic and treatment import. As such, treatment goals may be clarified based on the features associated with each symptom cluster. The various findings also underscore the need for further study of tic disorder symptomatology in youth. Over the past decade, the empirical literature on tic disorders has grown steadily out of what was once simply clinical observation (reviewed in Swain et al. 2007). The continued study of symptom dimensions in tic disorders, as in other psychiatric illnesses, will be of high priority (Fanous and Kendler 2005). Future studies may expand levels of analysis to include other 
possible dimensions of tic disorders, such as psychophysiological or neurobiological factors related to premonitory urge and genetic variables related to comorbidity, in a more comprehensive analysis of association and risk variables within this multifaceted symptom spectrum.

Open Access This article is distributed under the terms of the Creative Commons Attribution Noncommercial License which permits any noncommercial use, distribution, and reproduction in any medium, provided the original author(s) and source are credited.

\section{References}

Achenbach, T. M. (1991). Manual for the child behavior checklist/418 and 1991 child profile. Burlington: University of Vermont Department of Psychiatry.

Achenbach, T. M., \& Rescorla, L. (2001). Manual for the ASEBA school-age forms and profiles. Burlington: Research Center for Children, Youth, and Families.

Aldridge, J. W., Berridge, K. C., \& Rosen, A. R. (2004). Basal ganglia neural mechanisms of natural movement sequences. Canadian Journal of Physiology and Pharmacology, 82, 732-739.

Alsobrook, J. P., \& Pauls, D. L. (2002). A factor analysis of tic symptoms in Gilles de la Tourette's syndrome. American Journal of Psychiatry, 159, 291-296.

American Psychiatric Association. (2000). Diagnostic and statistical manual of mental disorders (4th ed.). Washington: American Psychiatric Association.

Banaschewski, T., Woerner, W., \& Rothenberger, A. (2003). Premonitory sensory phenomena and suppressibility of tics in Tourette syndrome: developmental aspects in youth and adolescents. Developmental Medicine and Child Neurology, 45, 700-703.

Breakwell, G. M., Hammond, S., Fife-Schaw, C., \& Smith, J. A. (2006). Research methods in psychology (3rd ed.). London: Sage Publications Ltd.

Conelea, C. A., \& Woods, D. W. (2008). The role of contextual factors in tic expression. Journal of Psychosomatic Research, 65, 487-496.

Fanous, A. H., \& Kendler, K. S. (2005). Genetic heterogeneity, modifier genes, and quantitative phenotypes in psychiatric illness: searching for a framework. Molecular Psychiatry, 10, 6-13.

Freeman, R. D., Fast, D. K., Burd, L., Kerbeshian, J., Robertson, M. M., \& Sandor, P. (2000). An international perspective on Tourette syndrome: Selected findings from 3, 500 individuals in 22 countries. Developmental Medicine and Child Neurology, 42, 436-447.

Grados, M. A., Riddle, M. A., Samuels, J. F., Liang, K.-Y., HoehnSaric, R., Bienvenu, O. J., et al. (2001). The familial phenotype of obsessive-compulsive disorder in relation to tic disorders: the Hopkins OCD family study. Biological Psychiatry, 50, 559-565.

Himle, M. B., Woods, D. W., Piacentini, J. C., \& Walkup, J. T. (2006). Brief review of habit reversal training for Tourette syndrome. Journal of Child Neurology, 21, 719-725.

Himle, M. B., Chang, S., Woods, D. W., Bunaciu, L., Pearlman, A., Buzzella, B., et al. (2007). Evaluating the contributions of ADHD, OCD, and tic symptoms in predicting functional competence in children with tic disorders. Journal of Developmental and Physical Disabilities, 19, 503-512.

Jagger, J., Prusoff, B. A., Cohen, D. J., Kidd, K. K., Carbonari, C. M., \& John, K. (1982). The epidemiology of Tourette's syndrome: a pilot study. Schizophrenia Bulletin, 8, 267-278.

Kaufman, J., Birmaher, B., Brent, D., \& Rao, U. (1997). Schedule for affective disorders and Schizophrenia for school-age childrenpresent and lifetime version (K-SADS-PL): initial reliability and validity data. Journal of the American Academy of Child and Adolescent Psychiatry, 36, 980-988.

Kuperman, S. (2002). Tic disorders in the adolescent. Adolescent Medicine, 13, 537-551.

Leckman, J. F. (2003). Phenomenology of tics and natural history of tic disorders. Brain Development, 25, S24-S28.

Leckman, J. F., Riddle, M. A., Hardin, M. T., Ort, S. L., Swartz, K. L., Stevenson, J., et al. (1989). The Yale Global Tic Severity Scale: initial testing of a clinician-rated scale of tic severity. Journal of the American Academy of Child and Adolescent Psychiatry, 28, $566-573$.

Leckman, J. F., King, R. A., \& Cohen, D. J. (1999). Tics and tic disorders. In J. F. Leckman \& D. J. Cohen (Eds.), Tourette's syndrome-tics, obsessions, compulsions: Developmental psychopathology and clinical care (pp. 23-42). New York: Wiley.

Leckman, J. F., Bloch, M. H., Scahill, L., \& King, R. A. (2006). Tourette syndrome: the self under siege. Journal of Child Neurology, 21, 642-649.

Mathews, C. A., Jang, K. L., Herrera, L. D., Lowe, T. L., Budman, C. L., Erenberg, G., et al. (2007). Tic symptom profiles in subjects with Tourette syndrome from two genetically isolated populations. Biological Psychiatry, 61, 292-300.

McMahon, W. M., Carter, A. S., Fredine, N., \& Pauls, D. L. (2003). Children at familial risk for Tourette's disorder: child and parent diagnoses. American Journal of Medical Genetics. Part B, Neuropsychiatric Genetics, 121B, 105-111.

Pauls, D. L., \& Hurst, C. R. (1981). Schedule for Tourette and other behavioral syndromes. New Haven: Yale University School of Medicine, Child Study Center.

Pauls, D. L., Towbin, K. E., Leckman, J. F., Zahner, G. E. P., \& Cohen, D. J. (1986). Gilles de la Tourette syndrome and obsessive compulsive disorder: evidence supporting a genetic relationship. Archives of General Psychiatry, 43, 1180-1182.

Reise, S. P., Waller, N. G., \& Comrey, A. L. (2000). Factor analysis and scale revision. Psychological Assessment, 12, 287-297.

Robertson, M. M., \& Cavanna, A. E. (2007). The Gilles de la Tourette Syndrome: a principal component factor analytic study of a large pedigree. Psychiatric Genetics, 17, 143-152.

Robertson, M. M., \& Eapen, V. (1996). The National Hospital Interview Schedule for the assessment of Gilles de la Tourette Syndrome and related behaviours. International Journal of Methods in Psychiatric Research, 6, 203-226.

Robertson, M. M., Althoff, R. R., Hafez, A., \& Pauls, D. L. (2008). Principal components analysis of a large cohort with Tourette syndrome. British Journal of Psychiatry, 193, 31-36.

Saccomani, L., Fabiana, V., Manuela, B., \& Giambattista, R. (2005). Tourette syndrome and chronic tics in a sample of children and adolescents. Brain Development, 27, 349-352.

Scahill, L., Riddle, M. A., McSwiggin-Hardin, M., Ort, S. I., King, R. A., Goodman, W. K., et al. (1997). Children's Yale-Brown ObsessiveCompulsive Scale: reliability and validity. Journal of the American Academy of Child and Adolescent Psychiatry, 36, 844-852.

Shapiro, A. K., Shapiro, E. S., Young, J. G., \& Feinberg, T. E. (1988). Gilles de la Tourette Syndrome (2nd ed.). New York: Raven.

Silverman, W., \& Albano, A. (1996). The anxiety disorders interview schedule for DSM-IV - child and parent versions. San Antonio: Graywind.

Silverman, W., Saavedra, L. M., \& Pina, A. (2001). Test-retest reliability of the anxiety symptoms and diagnoses with the Anxiety Disorders Interview Schedule for DSM-IV: child and parent versions. Journal of the American Academy of Child and Adolescent Psychiatry, 40, 937-943.

Storch, E. A., Murphy, T. K., Geffken, G. R., Soto, O., Sajid, M., Allen, P., et al. (2004). Psychometric evaluation of the Children's Yale-Brown Obsessive-Compulsive Scale. Psychiatry Research, 129, 91-98. 
Storch, E. A., Murphy, T. K., Geffken, G. R., Sajid, M., Allen, P., Roberti, J. W., et al. (2005). Reliability and validity of the Yale Global Tic Severity Scale. Psychological Assessment, 17, 486-491.

Storch, E. A., Murphy, T. K., Fernandez, M., Krishnan, M., Geffken, G. R., Kellgren, A. R., et al. (2007). Factor-analytic study of the Yale Global Tic Severity Scale. Psychiatry Research, 129, 231-237.

Swain, J. E., \& Leckman, J. F. (2003). Tourette's syndrome in children. Current Treatment Options in Neurology, 5, 299-308.

Swain, J. E., Scahill, L., Lombroso, P. J., King, R. A., \& Leckman, J. F. (2007). Tourette syndrome and tic disorders: a decade of progress. Journal of the American Academy of Child and Adolescent Psychiatry, 46, 947-968.

Tourette Syndrome Association International Consortium for Genetics. (1999). A complete genome screen in sib pairs affected by Gilles de la Tourette syndrome. American Journal of Human Genetics, 65, 1428-1426.

Walkup, J. T., Rosenberg, L. A., Brown, J., \& Singer, H. S. (1992). The validity of instruments measuring tic severity in Tourette's syndrome. Journal of the American Academy of Child and Adolescent Psychiatry, 31, 474-477.

Wolf, S. S., Jones, D. W., Knable, M. B., Gorey, J. G., Lee, K. S., Hyde, T. M., et al. (1996). Tourette syndrome: prediction of phenotypic variation in monozygotic twins by caudate nucleus D2 receptor binding. Science, 273, 1225-1227.

Wood, J., Piacentini, J., Bergman, R. L., McCracken, J., \& Barrios, V. (2002). Concurrent validity of the anxiety disorders section of the anxiety disorders interview schedule for DSM-IV: child and parent versions. Journal of Clinical Child and Adolescent Psychology, 31, 335-342.

Woods, D. W., Piacentini, J., Himle, M. B., \& Chang, S. (2005). Premonitory Urge for Tics Scale (PUTS): initial psychometric results and examination of the premonitory urge phenomenon in youths with tic disorders. Journal of Developmental and Behavioral Pediatrics, 26, 397-403.

Zhu, Y., Leung, K. M., Liu, P. Z., Zhou, M., \& Su, L. Y. (2006). Comorbid behavioural problems in Tourette's syndrome are positivity correlated with the severity of tic synptoms. The Australian and New Zealand Journal of Psychiatry, 40, 67-73. 